\title{
Acquired immunodeficiency syndrome in a patient with no known risk factors: a pathological study
}

\author{
AD BURT, ${ }^{*}$ GRACE SCOTT,† CAROLINE R SHIACH,‡ CG ISLES \\ From the *Department of Pathology and $¥$ Medical Unit, Western Infirmary, Glasgow G11 6NT, and \\ †University Department of Neuropathology, Institute of Neurological Sciences, Southern General Hospital, \\ Glasgow
}

SUMMARY We present the pathological findings in a case of acquired immunodeficiency syndrome (AIDS) in a patient with no known risk factor. Postmortem examination showed klebsiella lung abscess, generalised cytomegalovirus infection, cerebral toxoplasmosis, and a primary cerebral lymphoma. An additional feature was the presence of dilatation of the intrahepatic large bile ducts in association with an atypical distribution of cytomegalovirus. The relation between this case and previously reported cases of AIDS is discussed.

A syndrome of opportunistic infections and uncommon malignant tumours associated with altered $\mathrm{T}$ cell immune function has recently been described.' Several populations at risk have been identified-notably male homosexuals, ${ }^{1}$ intravenous drug abusers, ${ }^{2}$ and Haitian immigrants. ${ }^{3}$ We report the pathological findings in a 47 year old heterosexual man who fulfilled the clinical criteria for the diagnosis of AIDS $^{4}$ but who had none of the previously reported risk factors. Postmortem examination showed opportunistic infections similar to those found in patients with AIDS and a primary cerebral lymphoma. In addition, dilatation of the intrahepatic bile ducts was noted in the absence of distal obstruction. The role of cytomegalovirus infection in the pathogenesis of this lesion is discussed.

\section{Case report}

The patient was a 47 year old white man. He was heterosexual, denied drug abuse, and had no history of exposure to blood products but had recently worked in Equatorial Africa. He had previously had several episodes of malaria but had never taken malarial chemoprophylaxis. He presented to our hospital with an 18 month history of intermittent fever, weight loss, and latterly diarrhoea. Despite intensive investigation at several medical centres abroad, no cause had been found to explain these

Accepted for publication 9 November 1983 symptoms. Clinical examination showed fever (up to $39^{\circ} \mathrm{C}$ ), evidence of recent weight loss, right lower lobe pulmonary consolidation, and oral candidiasis. No organisms other than candida were isolated from numerous sputum, urine, and stool cultures. Serum IgG against cytomegalovirus was found on only one occasion at a titre of $1 / 64$, but other serological tests were negative. These included a screen for antibodies against Toxoplasma gondii, brucellosis, typhoid, syphilis, legionella, Q fever, and psittacosis, and fluorescent antibody tests for malaria, leishmaniasis, schistosomiasis, trypanosomiasis, and amoebiasis. Mantoux test $(1 / 1000)$ was negative, as was the screen for hepatitis B surface antigen. The peripheral blood lymphocyte count was depressed, but subpopulation studies were unfortunately not performed.

There was radiological evidence of dilatation of the intrahepatic bile ducts. Serum alkaline phosphatase activity was raised $(760 \mathrm{U} / \mathrm{l} ;$ normal $<130$ U/l). Despite intensive antimicrobial chemotherapy his fever continued and his general condition deteriorated. Latterly he developed focal neurological signs. A computed tomogram showed a mass in the temporal lobe. He subsequently became comatose and died.

\section{NECROPSY FINDINGS}

At necropsy both lungs showed basal consolidation with areas of haemorrhage and necrosis. A well demarcated abscess cavity ( $4 \mathrm{~cm}$ diameter) was present in the right lower lobe. Klebsiella pneumoniae and Candida albicans were subsequently isolated 


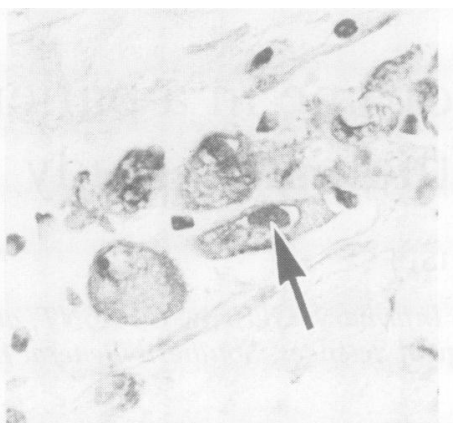

Fig. 1 Cytomegalovirus inclusion bodies (arrowed) within endothelial cells in a periductal venule. Red and yellow $\times 400$.

from the abscess. Microscopy of the adjacent lung parenchyma showed areas of necrosis with minimal accompanying inflammatory reaction. Occasional pneumocytes contained cytomegalovirus intranuclear inclusion bodies. There was no evidence of Pneumocystis carinii infection.

The liver weighed $2000 \mathrm{~g}$ but there was no splenomegaly (weight $200 \mathrm{~g}$ ). The large intrahepatic bile ducts were dilated as were the right and left hepatic ducts, but no gallstones were present within the extrahepatic biliary tract and no strictures were found. Histologically, the large intrahepatic ducts contained concretions composed of degenerate bile duct epithelial cells and bile crystals with occasional colonisation by Gram negative coliform bacteria. The connective tissue surrounding such ducts was thickened and swollen but there was minimal inflammatory infiltrate. The endothelial cells of periductal capillaries, arterioles, and venules con-

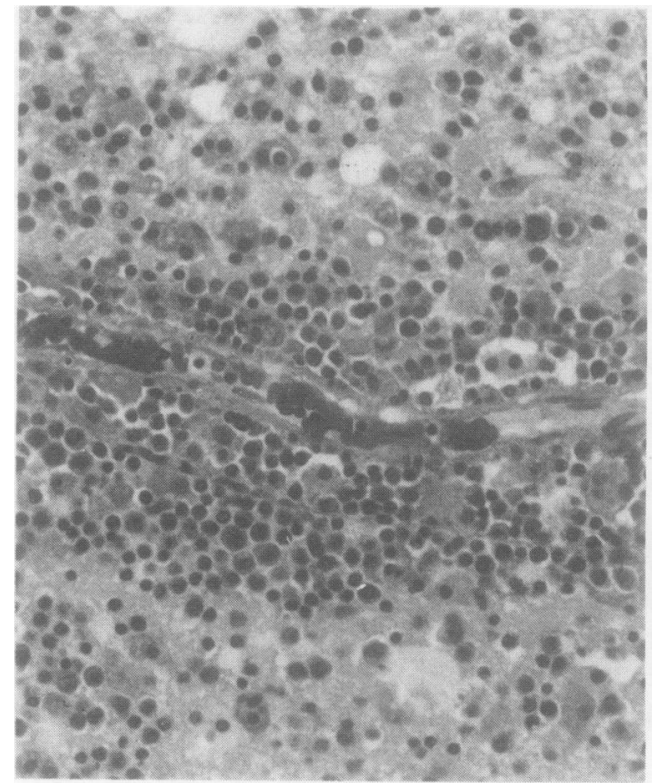

Fig. 3 Perivascular infiltration in brain by poorly differentiated lymphocytic lymphoma. Haematoxylin $\times 100$

tained large Feulgen positive intranuclear inclusion bodies. The presence of cytomegalovirus within such cells was subsequently shown by an indirect immunofluoresence technique (goat IgG anticytomegalovirus, Northwest Biomedical Laboratories). The endothelial cells were up to $30 \mu \mathrm{m}$ in diameter and in places occluded the lumina of periductal capillaries, arterioles, and venules (Fig. 1). Cytomegalovirus was also found within degenerate bile duct epithelial cells but was present within

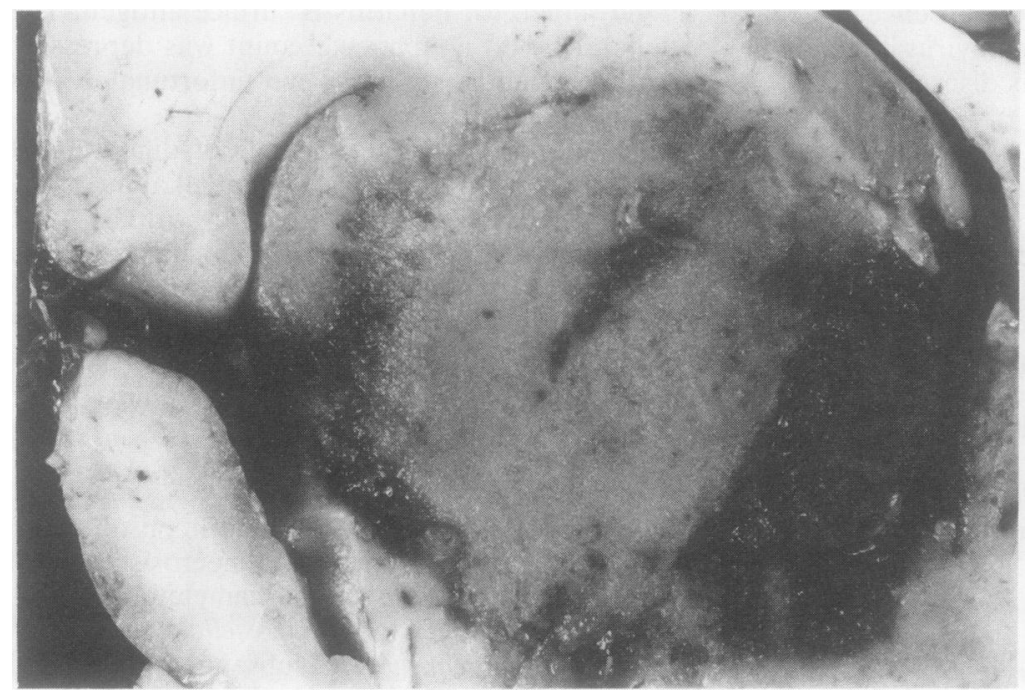

Fig. 2 Cerebral lymphoma centred on the left lentiform nucleus. 
only occasional hepatocytes. Bile duct injury was limited to the large bile ducts, the small and medium sized portal tracts being normal. There was no evidence of hepatocellular necrosis, and inflammatory changes within the hepatic acini were inconspicuous.

Cytomegalovirus infection was not restricted to the liver, inclusions being identified within endothelial cells in the submucosa of the gall bladder, duodenum, and sigmoid colon; within pancreatic acinar cells; and within thyroid epithelial cells.

The brain weighed $1300 \mathrm{~g}$. On coronal section a tumour mass was found centred on the left lentiform nucleus and measuring $5 \times 3 \times 3.5 \mathrm{~cm}$ (Fig. 2). The cut surfaces of the tumour had a finely granular appearance with areas of necrosis and haemorrhage. Tumour extended across the internal capsule and appeared to infiltrate the amygdaloid and subthalamic nuclei but spared the hypothalamus. There was a shift to the right of the midline structures of up to $6 \mathrm{~mm}$, but there was no evidence of internal herniation. Microscopy showed a diffuse, poorly differentiated, lymphocytic, non-Hodgkin's lymphoma. There was pronounced perivascular infiltration by malignant lymphoid cells along the Virchow Robin spaces into the thalamus and left insula (Fig. 3). No evidence of lymphoma was found in the brain stem or cerebellum, and there was no evidence of extracranial spread.

Immunohistochemical characterisation of the tumour using the peroxidase-antiperoxidase method $^{5}$ showed that the malignant cells did not

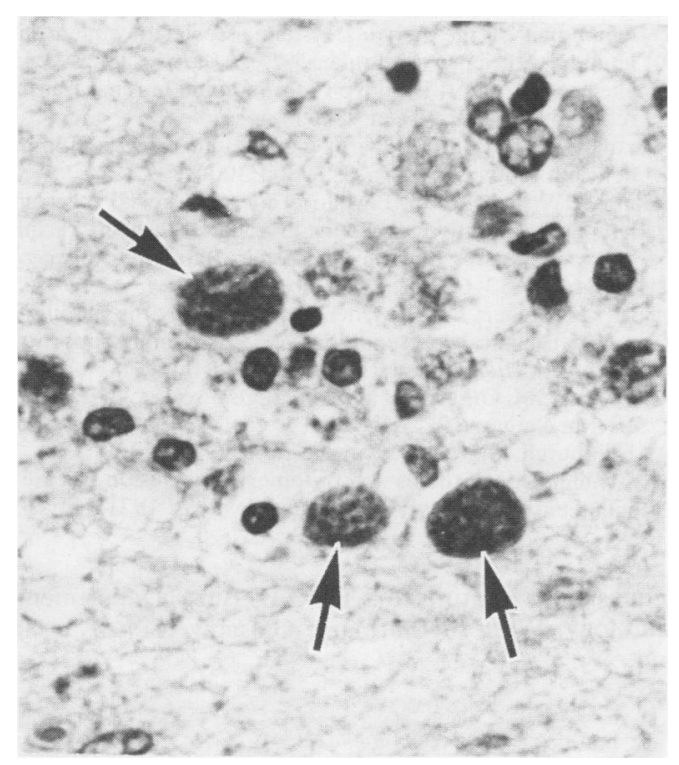

Fig. 4 Toxoplasma cysts (arrowed) within cerebral cortex (left parietal region) $\times 400$. contain immunoglobulin, $\alpha_{1}$-antichymotrypsin, or muramidase.

Numerous toxoplasma cysts were scattered throughout both cerebral hemispheres but not within the brain stem or cerebellum (Fig. 4). There was no apparent cellular reaction in relation to these parasites. No cytomegalovirus inclusion bodies were found in the brain.

\section{Discussion}

There is now much epidemiological evidence to suggest that the recently described acquired immunodeficiency syndrome in homosexual populations is the result of a communicable disease, though the nature of the putative transmissable agent remains uncertain. ${ }^{6}$ Cases with similar clinical manifestations have been reported in haemophiliacs, ${ }^{7}$ drug abusers, ${ }^{2}$ and Haitian immigrants. $^{3}$ It is uncertain whether the syndromes in these individual at risk groups have a common cause. Several sporadic cases have been described in which there has been no recognisable risk factor. Our patient had a history similar to cases included in the series of Brunet et al.$^{8}$ These workers described three previously healthy young heterosexual men who had recently travelled in Equatorial Africa and who had developed AIDS. Brunet et al suggested that the putative AIDS agent might be endemic in that area. The possibility that other immunosuppressive factors might be implicated in the pathogenesis of the syndrome in such individuals should, however, be considered. Exposure to malaria, for example, is known to compromise immune function. ${ }^{9}$

Our patient fulfilled the clinical criteria for the diagnosis of AIDS. ${ }^{4}$ Although lymphocyte subpopulations were not measured during life, indirect evidence of $T$ cell dysfunction was provided by the presence of lymphopenia and a negative Mantoux reaction. The range of opportunistic infection was similar to that described in previously reported cases of AIDS. Generalised cytomegalovirus infection and candidiasis are often encountered.' Luft et al ${ }^{10}$ described nine cases of toxoplasma encephalitis arising in Haitian immigrants with AIDS, though details of the neuropathological findings were not given. The typical histological appearances of cerebral toxoplasmosis are those of a necrotising granulomatous reaction around the toxoplasma cysts and endozoites. " Evidence of such a host response was absent in our case. The large number and widespread distribution of the toxoplasma cysts, however, suggest, that this was a recently acquired infection. While this may reflect an early stage of the disease, it may represent a further manifestation of 
the inability of patients with AIDS to mount a granulomatous response. ${ }^{12}$ Similar morphological findings have been reported in other immunocompromised patients with toxoplasmosis. ${ }^{13}$

Two cases of primary central nervous system lymphoma arising in homosexual patients with AIDS have previously been reported..$^{14}$ There is a well recognised increased incidence of primary central nervous system lymphoma in other immunocompromised patients, as shown by renal transplant recipients receiving immunosuppressive treatment. ${ }^{\text {is }}$ The pathogenesis of these comparatively rare tumours is uncertain, though lymphotropic DNA viruses such as Epstein-Barr virus have been implicated. ${ }^{16}$ There is evidence that many of the central nervous system lymphomas arising in renal transplant recipients may initially be polyclonal $\mathrm{B}$ cell tumours. ${ }^{17}$ By contrast, our case is similar to one of the cases reported by Snider $e a^{14}$ in that it was a poorly differentiated lymphocytic lymphoma expressing neither B cell nor histiocyte markers. It is uncertain whether this reflects a different pathogenesis.

The hepatic lesions that we have described here have not been previously reported in AIDS. Distal bile duct obstruction was excluded, and it is suggested that the dilatation of the intrahepatic ducts may have been the result of ischaemic damage by occlusion of the periductal capillaries, venules, and arterioles by cytomegalovirus infected endothelial cells. Such a mechanism has been proposed for cytomegalovirus cutaneous vasculitis. ${ }^{18}$ The distribution of cytomegalovirus within the liver is unusual. In adults the virus is most commonly associated with a lobular hepatitis, which may be granulomatous. ${ }^{19}$ Damage to bile ducts is seen in cytomegalovirus infection of the fetus, but this affects mainly ducts. ${ }^{20}$ We suggest that the atypical distribution of cytomegalovirus within the liver in this case may have been related to the altered immune state of the patient.

We thank Dr SG Ball, Professor RNM MacSween, and Professor DI Graham for their helpful advice and criticism, and we are indebted to Dr JF Boyd for performing the cytomegalovirus indirect immunofluorescence tests.

\section{References}

' Gottlieb MS, Schroff R, Schanker HM, et al. Pneumocystis carinii pneumonia and mucosal candidiasis in previously healthy homosexual men; evidence of a new acquired cellular immunodeficiency. $N$ Engl J Med 1981;305:1425-30.

${ }^{2}$ Masur H, Michelis MA, Greene JB, et al. An outbreak of community-acquired Pneumocystis carinii pneumonia: initial manifestations of cellular immune dysfunction. $N$ Engl $J$ Med 1981;305:1431-8.

${ }^{3}$ Vieira J, Frank E, Spira TJ, Landesman SH. Acquired immune deficiency in Haitians. Opportunistic infections in previously healthy Haitian immigrants. $N$ Engl J Med 1983;308:125-9.

4 Update on AIDS-United States. Morbid Mortal Week Rep 1982;31:507-8.

s Sternberger LA, Hardy PN, Cuculis JJ, Meyer NG. The unlabelled antibody enzyme method of immunohistochemistry: preparation and properties of soluble antigen-antibody complex (horseradish peroxidase-anti-horseradish peroxidase) and its use in the identification of spirochaetes.J Histochem Cytochem 1970;18:315-33.

- Waterson AP. Acquired immune deficiency syndrome. Br Med J 1983;286:743-6.

7 Poon MC, Lauday A, Prasthofer EF, Stagno S. Acquired immunodeficiency syndrome with Pneumocystis carinii pneumonia and Mycobacterium avium-intracellulare infection in a previously healthy patient with classic haemophilia. Ann Int Med 1983;98:287-90.

${ }^{8}$ Brunet JB, Bouvet E, Leibowitch J, et al. Acquired immunodeficiency syndrome in France. Lancet 1983;i:700-1.

9 Greenwood BM, Bradley-More AM, Palit A, Bryceson ADM. Immunosuppression in children with malaria. Lancet 1972;i:169.

${ }^{10}$ Luft BJ, Cowley F, Remington JS, et al. Outbreak of centralnervous system toxoplasmosis in Western Europe and North America. Lancet 1983;i:781-3.

"Adams JH. Parasitic and fungal infections of the nervous system. In: Blackwood W, Corsellis JAN, eds. Greenfield's neuropathology. London: Edward Arnold, 1976:269-91.

${ }^{12}$ Cohen RJ, Samoszuk MK, Busch D, Lagios M. Occult infections with $\mathrm{M}$ Intracellulare in bone-marrow biopsy specimens from patients with AIDS. $N$ Engl J Med 1983;308:1475-6.

${ }^{13}$ Ghatak NR, Sawyer DR. A morphologic study of opportunistic cerebral toxoplasmosis. Acta Neuropathol (Berlin) 1978; 42:217-21.

${ }^{14}$ Snider WD, Simpson DM, Aronyk KE, Neilsen SL. Primary lymphoma of the nervous system associated with acquired immune-deficiency syndrome. $N$ Engl J Med 1983;308:45.

${ }^{15}$ Schneck SA, Penn I. De novo brain tumours in renal transplant recipients. Lancet 1971;i:983-6.

${ }^{16}$ Purtillo DT. Epstein Barr virus induced oncogenesis in immune deficient individuals. Lancet 1980;i:300-3.

${ }^{17}$ Hanto DW, Frizzera G, Purtillo DT, et al. Clinical spectrum of lymphoproliferative disorders in renal transplant recipients and evidence for the role of Epstein Barr virus. Cancer Res 1981;41:4253-61.

${ }^{18}$ Curtis JL, Egbert BM. Cutaneous cytomegalovirus vasculitis: an unusual clinical presentation of a common opportunistic pathogen. Hum Pathol 1982;13:1138-41.

${ }^{19}$ Edington GM. Other viral and infectious diseases. In: MacSween RNM, Anthony PP, Scheuer PJ, eds. Pathology of the liver. Edinburgh: Churchill Livingstone, 1979:232-47.

${ }^{20}$ Altschuler G, McAdams AJ. Cytomegalic inclusion disease in a $\mathrm{C}$ nineteen-week fetus. Am J Obstet Gynaecol 1971;111:295-8.

Requests for reprints to: $\mathrm{Dr}$ AD Burt, Department of Pathology, Western Infirmary, Glasgow G11 6NT, Scotland. 